ALEA, Lat. Am. J. Probab. Math. Stat. 18, 963-983 (2021)

DOI: 10.30757/ALEA.v18-35

\title{
Ergodicity of the KPZ Fixed Point
}

\section{Leandro P. R. Pimentel}

Institute of Mathematics, Federal University of Rio de Janeiro

149 Av. Athos da Silveira Ramos,

Postal Code 21941-909,

Rio de Janeiro, RJ, Brazil.

E-mail address: leandro@im.ufrj.br

URL: https://sites.google.com/site/leandroprpimentel/

\begin{abstract}
In this paper we consider the Kardar-Parisi-Zhang (KPZ) fixed point $\left(\mathfrak{h}_{t}, t \geq 0\right)$ (Corwin et al., 2015; Matetski et al., 2016) and prove that, for suitable initial conditions, $\mathfrak{h}_{t}(x)-\mathfrak{h}_{t}(0)$ converges to a two-sided Brownian motion with zero drift and diffusion coefficient 2 , as $t \rightarrow \infty$. The heart of the proof is the coupling method, that allows us to compare the TASEP height function started from a perturbation of density $1 / 2$ with its invariant counterpart which, under $\mathrm{KPZ}$ scaling, turns into uniform estimates for the KPZ fixed point
\end{abstract}

\section{Introduction and Main Result}

In $d+1$ stochastic growth models the object of interest is a height function, $h(x, t)$ for $x \in \mathbb{R}^{d}$ (space) and $t \geq 0$ (time), whose evolution is described by a random mechanism. For fairly general growth models one has a deterministic macroscopic shape for the $h(x, t)$ and its fluctuations, under proper space-time scaling, are expected to be characterized by a universal distribution. The Kardar-Parisi-Zhang (KPZ) fixed point is a Markov process introduced by Corwin, Quastel and Remenik (Corwin et al., 2015) and Matetski, Quastel and Remenik (Matetski et al., 2016) that gives the limit fluctuations of a wide class of growth models with $d=1$ that possesses a local slope dependent growth rate and a smoothing mechanism, combined with space-time random forcing with rapid decay of correlations. The KPZ equation (Kardar et al., 1986)

$$
\frac{\partial h}{\partial t}=\frac{1}{2}\left(\frac{\partial h}{\partial x}\right)^{2}+\frac{\partial^{2} h}{\partial^{2} x}+\xi,
$$

Received by the editors December 18th, 2019; accepted October 27th, 2020.

2010 Mathematics Subject Classification. 60K35, 82C22.

Key words and phrases. KPZ Fixed Point, Brownian Motion, Growth Models, Totally Asymmetric Simple Exclusion Process.

Research supported by CNPQ grants $421383 / 2016-0$ and $302830 / 2016-2$ and by the FAPERJ grant E-26/203.048/2016. 
where $\xi$ is a space-time white noise, is a canonical example of such a growth model, providing its name to the universality class (Amir et al., 2011; Borodin et al., 2015). It is conjectured that for such models

$$
h\left(a n^{2 / 3} x, n t\right) \stackrel{\text { dist. }}{\sim} b t n+c n^{1 / 3} \mathfrak{h}_{t}(x),
$$

for some model dependent constants $a, b, c \in \mathbb{R}$, where $\mathfrak{h}_{t}(x)$ is a universal spacetime process, called the KPZ-fixed point.

Another canonical example of KPZ fluctuations is given by the height function associated to the totally asymmetric simple exclusion process (TASEP), where the limiting behaviour was stablished initially by Johansson $(2000,2003)$. In the past twenty years there has been a significant amount of improvements of the theory. The exact statistics for different initial interface profiles, resulting in different types of Airy processes, were computed using the TASEP height function and its connection with integrable probability (Baik et al., 2010; Baik and Rains, 2000; Corwin et al., 2016; Prähofer and Spohn, 2002). Recently, a unifying approach based on a previous work of Sasamoto and coauthors (Borodin et al., 2007; Sasamoto, 2005) was developed by Matetski, Quastel and Remenik (Matetski et al., 2016). They derived a Fredholm determinant formula for the finite dimensional distributions of the TASEP height function, and proved that this formula led to the computation of the transition probabilities of the KPZ fixed point.

We say that a function $\mathfrak{h}: \mathbb{R} \rightarrow \mathbb{R} \cup\{-\infty\}$ is upper semicontinuous if its hypograph $\mathfrak{H}:=\{(x, y): y \leq \mathfrak{h}(x)\}$ is closed in $\mathbb{R} \times(\mathbb{R} \cup\{-\infty\})$, where the distance $d$ between $\left(x_{1}, y_{1}\right)$ and $\left(x_{2}, y_{2}\right)$ is defined as $\left|x_{1}-x_{2}\right|+\left|e^{y_{1}}-e^{y_{2}}\right|$. This distance is appropriate for the evolution of the KPZ fixed point because it allows continuity at time zero for an initial profile that has values $-\infty$. The Hausdorff distance between two subsets $A$ and $B$ of $\mathbb{R} \times(\mathbb{R} \cup\{-\infty\})$ is defined as

$$
d_{H}(A, B):=\max \{d(A, B), d(B, A)\},
$$

where

$$
d(A, B):=\sup _{\left(x_{1}, y_{1}\right) \in A} \inf _{\left(x_{2}, y_{2}\right) \in B} d\left(\left(x_{1}, y_{1}\right),\left(x_{2}, y_{2}\right)\right) .
$$

The evolution of the KPZ fixed point takes place on the space UC of upper semicontinuous functions $\mathfrak{h}: \mathbb{R} \rightarrow \mathbb{R} \cup\{-\infty\}$ such that $\mathfrak{h}(x)<C(1+|x|)$ for some $C>0$. The topology of local convergence in UC can be defined in terms of the Hausdorff distance between hypographs: given $a>0$ and $\epsilon>0$, we say that $\mathfrak{h}_{1}, \mathfrak{h}_{2} \in \mathrm{UC}$ restricted to $[-a, a]$ are $\epsilon$-close if $d_{H}\left(\mathfrak{H}_{1}^{a}, \mathfrak{H}_{2}^{a}\right)<\epsilon$, where $\mathfrak{H}_{i}^{a}$ denotes the hypograph of $\mathfrak{h}_{i}$ restricted to $[-a, a]$, for $i=1,2$. We also consider the the space $C$ of continuous functions $\mathfrak{h}: \mathbb{R} \rightarrow \mathbb{R}$, such that $\mathfrak{h}(x)<C(1+|x|)$ for some $C>0$, endowed with uniform norm on compact sets

$$
\|\mathfrak{h}\|_{\infty, a}:=\sup _{[-a, a]}|\mathfrak{h}(x)|,
$$

where $a>0$. The topology of local convergence in UC when restricted to $\mathrm{C}$ is the topology of uniform convergence on compact sets.

The family composed by sets of the form

$$
\mathrm{A}_{\mathfrak{g}}:=\{\mathfrak{h} \in \mathrm{UC}: \mathfrak{h}(x) \leq \mathfrak{g}(x), \forall x \in \mathbb{R}\},
$$

where $-\mathfrak{g} \in \mathrm{UC}$ ( $\mathfrak{g}$ is a lower semicontinuous function), defines a generating family of the Borel subsets of UC. Let $\mathfrak{h}_{t} \equiv \mathfrak{h}_{t}(\cdot ; \mathfrak{h})$ denote the KPZ fixed point starting at 
$\mathfrak{h}_{0}=\mathfrak{h}$. For a fixed time $t>0$ the distribution of $\mathfrak{h}_{t}(\cdot ; \mathfrak{h})$ is determined by

$$
\mathbb{P}\left(\mathfrak{h}_{t}(\cdot ; \mathfrak{h}) \in \mathrm{A}_{\mathfrak{g}}\right)=\operatorname{det}\left(\mathbf{I}-\mathbf{K}_{t, \mathfrak{h}, \mathfrak{g}}\right)_{L^{2}(\mathbb{R})},
$$

where $-\mathfrak{g} \in$ UC. For a description of the integral operator $\mathbf{K}_{t, \mathfrak{h}, \mathfrak{g}}$ in terms of $\mathfrak{h}$ and $\mathfrak{g}$ the author directs the reader to Matetski et al. (2016). The determinant on the right hand side is a Fredholm determinant on the Hilbert space $L^{2}(\mathbb{R})$, and $\mathbf{I}$ is the identity:

$$
\operatorname{det}\left(\mathbf{I}-\mathbf{K}_{t, \mathfrak{h}, \mathfrak{g}}\right)_{L^{2}(\mathbb{R})}:=\sum_{n=0}^{\infty} \frac{(-1)^{n}}{n !} \int_{\mathbb{R}^{n}} \operatorname{det}\left(K\left(x_{i}, x_{j}\right)\right)_{i, j=1}^{n} d x_{1} \cdots d x_{n},
$$

where $K(x, y)$ is the kernel of the integral operator $\mathbf{K}_{t, \mathfrak{h}, \mathfrak{g}}$. From this formula one can recover several of the classical Airy processes by starting with special profiles for which the respective operators $K$ are explicit (see Section 4.4 of Matetski et al., 2016). For instance, the Airy ${ }_{2}$ process $\mathcal{A}(\cdot)=\mathfrak{h}(\cdot ; \mathfrak{d})$ is defined by taking the initial profile $\mathfrak{h}=\mathfrak{d}$ where $\mathfrak{d}(0)=0$ and $\mathfrak{d}(z)=-\infty$ for all $z \neq 0$.

The KPZ fixed point satisfies some fundamental symmetries which are expressed below for fixed $t>0$ in terms of distributional equalities as processes in UC (Matetski et al., 2016).

(i) 1-2-3 Scaling: $S_{\gamma^{-1}} \mathfrak{h}_{\gamma^{-3} t}\left(\cdot ; S_{\gamma} \mathfrak{h}\right) \stackrel{\text { dist. }}{=} \mathfrak{h}_{t}(\cdot ; \mathfrak{h})$, where

$$
S_{\gamma} \mathfrak{h}(x):=\gamma^{-1} \mathfrak{h}\left(\gamma^{2} x\right), \text { for } \gamma>0 \text {. }
$$

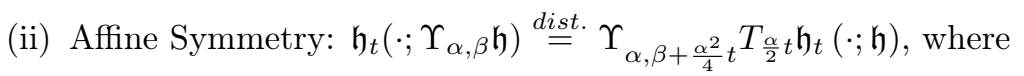

$$
\Upsilon_{\alpha, \beta} \mathfrak{h}(x):=\beta+\alpha x+\mathfrak{h}(x) \text { and } T_{z} \mathfrak{h}(x):=\mathfrak{h}(z+x) .
$$

(iii) Space Stationarity: $T_{z} \mathfrak{h}_{t}\left(\cdot ; T_{-z} \mathfrak{h}\right) \stackrel{\text { dist. }}{=} \mathfrak{h}_{t}(\cdot, \mathfrak{h})$.

We note that, by the 1-2-3 scaling (i) with $\gamma=t^{1 / 3}>0$, we have that

$$
\mathfrak{h}_{t}(\cdot ; \mathfrak{h}) \stackrel{\text { dist. }}{=} S_{t^{-1 / 3}} \mathfrak{h}_{1}\left(\cdot ; S_{t^{1 / 3}} \mathfrak{h}\right)
$$

Another important symmetry is related to time invariance: let $\mathfrak{b}$ be a two-sided Brownian motion with diffusion coefficient 2 , then

$$
\Delta \mathfrak{h}_{t}(\cdot ; \mathfrak{b}) \stackrel{\text { dist. }}{=} \mathfrak{b}(\cdot) \text { for all } t \geq 0, \text { where } \Delta \mathfrak{h}(x):=\mathfrak{h}(x)-\mathfrak{h}(0)
$$

Combining this with (ii) (affine invariance), one sees that the drifted Brownian motion $\Upsilon_{0, \alpha} \mathfrak{b}$ is also invariant. The aim of this article is to prove convergence of $\Delta \mathfrak{h}_{t}$ to $\mathfrak{b}$, as $t \rightarrow \infty$, for suitable initial conditions (ergodicity).

The study of local space regularity in the KPZ universality class was initiated by Hägg (2008), where it was proved local finite-dimensional convergence of the Airy 2 process to Brownian motion, using asymptotic estimates related to a determinantal structure similar to (1.1). This result was extended to functional convergence in Cator and Pimentel (2015), by means of the coupling method. Using kernel estimates for the operator in (1.1), Matetski, Quastel and Remenik (Matetski et al., 2016) proved that for every $\mathfrak{h} \in \mathbf{U C}$ and $t>0, \mathfrak{h}_{t}(\cdot ; \mathfrak{h})$ has Hölder $1 / 2$ - regularity in space, in particular $\mathfrak{h}_{t}(\cdot ; \mathfrak{h}) \in \mathbf{C}$, and that

$$
S_{\sqrt{\epsilon}} \Delta \mathfrak{h}_{t}(x)=\epsilon^{-1 / 2}\left(h_{t}(\epsilon x ; \mathfrak{h})-h_{t}(0 ; \mathfrak{h})\right)
$$

converges to $\mathfrak{b}$, as $\epsilon \rightarrow 0$, in terms of finite dimensional distributions. Functional convergence was proved by Pimentel (2018) for several versions of Airy processes, 
and stronger forms of local Brownian behaviour were proved by Corwin and Hammond (2014) and Hammond (2019). From the 1-2-3 scaling (1.2) and (1.3), the long time behaviour of $\Delta \mathfrak{h}_{t}$ can be rewriten in terms of the local behaviour of the process at time $t=1$,

$$
\Delta \mathfrak{h}_{t}(\cdot ; \mathfrak{h}) \stackrel{\text { dist. }}{=} S_{\sqrt{\epsilon}} \Delta \mathfrak{h}_{1}\left(\cdot ; S_{\sqrt{\epsilon^{-1}}} \mathfrak{h}\right), \text { with } \epsilon=t^{-2 / 3}
$$

which allows one to obtain ergodicity (in terms of finite dimensional distributions) from the local convergence to Brownian motion with zero drift, as soon as $S_{\sqrt{\epsilon^{-1}}} \mathfrak{h}$ has a limit.

Recently there has been considerable developments in describing the space-time structure of the KPZ fixed point in terms of a variational formula (Corwin et al., 2016; D. Dauvergne and Virág, 2018; Ferrari and Occelli, 2018; Matetski et al., 2016) involving the Airy $_{2}$ process: for fixed $x \in \mathbb{R}$,

$$
\mathfrak{h}_{t}(x ; \mathfrak{h}) \stackrel{\text { dist. }}{=} \sup _{z \in \mathbb{R}}\left\{\mathfrak{h}(z)+t^{1 / 3} \mathcal{A}\left(z t^{-2 / 3}\right)-\frac{(z-x)^{2}}{t}\right\} \text {. }
$$

(See Theorem 4.18 and Remark 4.19 in Matetski et al., 2016.) In this paper we rely on (1.5) and use the coupling method applied to a last-passage percolation discrete approximation of $\mathfrak{h}_{t}$ to prove long time convergence in probability. As it is indicated in (1.4), we need to assume some control of $S_{\gamma} \mathfrak{h}$ (1.2) for large values of $\gamma$, which is expressed in (1.6) below.

Theorem 1.1. Assume there exist $\gamma_{0}>0$ and $\psi(r)$ such that for all $\gamma>\gamma_{0}$ and $r \geq 1$

$$
\mathbb{P}\left(S_{\gamma} \mathfrak{h}(x) \leq r|x|, \forall|x| \geq 1\right) \geq 1-\psi(r), \text { where } \lim _{r \rightarrow \infty} \psi(r)=0 .
$$

Let $a>0$ and $t \geq a^{3 / 2}$. Under assumption (1.6), there exists a coupling between $\mathfrak{h}_{t}(\cdot ; \mathfrak{h})$ and $\mathfrak{h}_{t}(\cdot ; \mathfrak{b})$, where $\mathfrak{h}$ and $\mathfrak{b}$ are sampled independently at $t=0$, and a function $\theta(\delta)$ such that

$$
\mathbb{P}\left(\left\|\Delta \mathfrak{h}_{t}(\cdot ; \mathfrak{h})-\Delta \mathfrak{h}_{t}(\cdot ; \mathfrak{b})\right\|_{\infty, a}>\eta \sqrt{a}\right) \leq \theta\left(a t^{-2 / 3}\right)+\frac{\left(a t^{-2 / 3}\right)^{1 / 4}}{\eta}, \forall \eta>0,
$$

where $\lim _{\delta \rightarrow 0} \theta(\delta)=0$.

Remark 1.2. The proof of Theorem 1.1 is based on a coupling that requires assumption (1.6) to prove tightness of transversal fluctuations of a maximal path around the origin in the (exponential) last-passage percolation model under the $2 / 3$ scaling (Proposition 3.6). The function $\theta$ might depend on $\psi$, but not on the parameters $a, t>0$, and its behaviour is also connected to transversal fluctuations of a maximal path. We note that the drifted Brownian motion $\Upsilon_{0, \alpha}$, with $\alpha \neq 0$, is also invariant and it certainly does not satisfy Assumption (1.6). In this case, by symmetry (ii), transversal fluctuations drift away from the origin with speed $\alpha / 2$ as $t \rightarrow \infty$.

As a consequence of Theorem 1.1, one can let $a=a_{t}$ (e.g. $a_{t}=t^{\kappa}$ for $\left.\kappa \in(0,2 / 3)\right)$ and still get convergence to equilibrium under proper rescaling.

Corollary 1.3. Under assumption (1.6), if $\lim _{t \rightarrow \infty} a_{t} t^{-2 / 3}=0$ then

$$
\lim _{t \rightarrow \infty} \mathbb{P}\left(\left\|\Delta \mathfrak{h}_{t}(\cdot ; \mathfrak{h})-\Delta \mathfrak{h}_{t}(\cdot ; \mathfrak{b})\right\|_{\infty, a}>\eta \sqrt{a_{t}}\right)=0, \quad \text { as } t \rightarrow \infty \text {. }
$$

In particular, since $\Delta \mathfrak{b}_{t}$ is invariant under diffusive scaling,

$$
\lim _{t \rightarrow \infty} S_{\sqrt{a_{t}}} \Delta \mathfrak{h}_{t} \stackrel{\text { dist. }}{=} \mathfrak{b} .
$$




\section{The TASEP Growth Process and Last-Passage Percolation}

The totally asymmetric simple exclusion process (TASEP) is a Markov process $\left(\eta_{t}, t \geq 0\right)$ with state space $\{0,1\}^{\mathbb{Z}}$. When $\eta_{t}(k)=1$, we say that site $x$ is occupied by a particle at time $t$, and it is empty (hole) if $\eta_{t}(k)=0$. Particles jump to the neighbouring right site with rate 1 provided that the site is empty (the exclusion rule). Given an initial profile of particles $\eta=\eta_{0} \in\{0,1\}^{\mathbb{Z}}$, we associate a label $j \in \mathbb{Z}$ to each particle, where we follow the standard ordering of particle locations from right to left as

$$
\cdots<X^{\eta}(2)<X^{\eta}(1)<0 \leq X^{\eta}(0)<X^{\eta}(-1)<\cdots .
$$

Placing particles at $\pm \infty$ enable us to start the process with right- or left-finite data with no change of notation, and the particles placed at $\pm \infty$ playing no rule in the evolution of the process. The ordering in (2.1) holds for particles located at positions $k \in \mathbb{Z}$. For instance, if we have

$$
\cdots, \eta(-2)=1, \eta(-1)=0, \eta(0)=0, \eta(1)=1, \eta(2)=0 \cdots,
$$

then the order of particles is given by $\cdots<X^{\eta}(1)=-2<X^{\eta}(0)=1<\cdots$. Another example is $\eta(k)=1$ if $k \leq 0$ and $\eta(k)=0$ if $k>0$. Then $X^{\eta}(j)=-j$ for all $j \geq 0$ and $X^{\eta}(j)=\infty$ for all $j<0$. We denote $X_{t}^{\eta}(j)$ the position of particle $j$ at time $t \geq 0$, where $X_{0}^{\eta}(j)=X^{\eta}(j)$. Due to the exclusion rule, the ordering of particles is preserved for all times: $X_{t}^{\eta}(j+1)<X_{t}^{\eta}(j)$.

For an initial $\eta \in\{0,1\}^{\mathbb{Z}}$, let $N_{t}^{\eta}$ denote the total number of particles which jumped from site 0 to site 1 during the time interval $[0, t]$. The TASEP growth model is represented by a height function $h_{t}^{\eta}: \mathbb{Z} \rightarrow \mathbb{Z}$, defined for each $t \geq 0$ as

$$
h_{t}^{\eta}(k)= \begin{cases}2 N_{t}^{\eta}+\sum_{j=1}^{k}\left(1-2 \eta_{t}(j)\right) & \text { for } k \geq 1 \\ 2 N_{t}^{\eta} & \text { for } k=0 \\ 2 N_{t}^{\eta}-\sum_{j=k+1}^{0}\left(1-2 \eta_{t}(j)\right) & \text { for } k \leq-1 .\end{cases}
$$

The correspondence between particle positions and height profile is as follows (see e.g. Baik et al., 2010):

$$
\mathbb{P}\left(\cap_{k=1}^{l}\left\{X_{j_{k}}^{\eta}(t) \geq i_{k}-j_{k}\right\}\right)=\mathbb{P}\left(\cap_{k=1}^{l}\left\{h_{t}^{\eta}\left(i_{k}-j_{k}\right) \geq i_{k}+j_{k}\right\}\right) .
$$

We extend $h_{t}^{\eta}$ to a continuous function $h_{t}^{\eta}: \mathbb{R} \rightarrow \mathbb{R}$ by linear interpolation of its values at integer numbers. For a given sequence of initial profiles $\left(\eta^{(n)}, n \geq 1\right)$, let $^{1}$

$$
\mathfrak{h}_{t, n}\left(x ; \eta^{(n)}\right):=\frac{t n-h_{2 t n}^{\eta^{(n)}}\left(2 x n^{2 / 3}\right)}{n^{1 / 3}}, \text { for } x \in \mathbb{R} .
$$

Matetski, Quastel and Remenik (Matetski et al., 2016) proved that if

$$
\lim _{n \rightarrow \infty} \mathfrak{h}_{0, n}\left(\cdot ; \eta^{(n)}\right) \stackrel{\text { dist. }}{=} \mathfrak{h}(\cdot)
$$

then

$$
\lim _{n \rightarrow \infty} \mathfrak{h}_{t, n}\left(\cdot ; \eta^{(n)}\right) \stackrel{\text { dist. }}{=} \mathfrak{h}_{t}(\cdot ; \mathfrak{h}) .
$$

While weak convergence (2.4) is considered with respect to the topology of local convergence in UC, for $t>0$ (2.5) holds with respect to the uniform topology of local convergence in C. We note that for any deterministic $\mathfrak{h} \in \mathrm{UC}$, with $\mathfrak{h}(0)=$

\footnotetext{
${ }^{1}$ In Matetski et al. (2016) they considered the growth interface moving downwards and the scaling factor $\epsilon \rightarrow 0$, while we are considering it moving upwards and setting $\epsilon=n^{-2 / 3}$.
} 
0 , one can construct a sequence of initial particle configurations $\eta^{(n)}$ such that $\mathfrak{h}_{0, n}(\cdot)=\mathfrak{h}_{0, n}\left(\cdot ; \eta^{(n)}\right) \rightarrow \mathfrak{h}(\cdot)$ in UC. Assumption (2.4) restricts the study of the scaling limit to perturbations of density $p=1 / 2$, where one has a limit at time zero under diffusive scaling. For instance, if $\eta^{(n)}(k)$ for $k \in \mathbb{Z}$ are i.i.d. with $\left|\mathbb{P}\left[\eta^{(n)}(k)=1\right]-1 / 2\right| \sim n^{-1 / 3}$ then $\mathfrak{h}_{0, n}\left(\cdot ; \eta^{(n)}\right)$ converges to a drifted two-sided Brownian motion. As observed by Matetski, Quastel and Remenik (Matetski et al., 2016), one could obtain the same scaling limit by perturbing off any density $p \in$ $(0,1)$ at time zero, and observing the interface profile in an appropriate moving frame.

Given a particle profile $\eta \in\{0,1\}^{\mathbb{Z}}$ we order particles as in (2.1) and holes from left to right as

$$
\cdots<Y^{\eta}(-1)<Y^{\eta}(0)<0 \leq Y^{\eta}(1)<Y^{\eta}(2) \cdots .
$$

In our example (2.2), the order of particles and holes is given by

$$
\cdots<X^{\eta}(1)=-2<Y^{\eta}(0)=-1<Y^{\eta}(1)=0<X^{\eta}(0)=1<Y^{\eta}(2)=2<\cdots .
$$

Notice that the labels of the holes in front of the particle $X^{\eta}(j)$ are given by $i>U^{\eta}(j)$ where

$$
U^{\eta}(j):=X^{\eta}(j)+j .
$$

In (2.2), we have that $U^{\eta}(1)=-2+1=-1$ and the holes in front of particle $X^{\eta}(1)$ are $Y^{\eta}(0), Y^{\eta}(1), \cdots$. Given $j \in \mathbb{Z}$ and $i>U^{\eta}(j)$ let

$$
G^{\eta}(i, j):=\text { the time when hole } i \text { interchanges position with particle } j \text {. }
$$

For convenience, we set $G^{\eta}(i, j):=-\infty$ if $i \leq U^{\eta}(j)$. Now, given $i>U^{\eta}(j)$ we write $i=n+U^{\eta}(j)$ for some $n \geq 1$. Then $G^{\eta}(i, j) \leq t$ if and only if $X_{t}^{\eta}(j)-X_{0}^{\eta}(j) \geq n$ (that is, particle $j$ made at least $n$ steps to the right by time $t$ ). On the other hand,

$$
X_{t}^{\eta}(j)-X_{0}^{\eta}(j) \geq n \Leftrightarrow X_{t}^{\eta}(j) \geq X^{\eta}(j)+n=i-j,
$$

and hence

$$
G^{\eta}(i, j) \leq t \Leftrightarrow X_{t}^{\eta}(j) \geq i-j
$$

By (2.3) and (2.7),

$$
\mathbb{P}\left(\cap_{k=1}^{l}\left\{G^{\eta}\left(i_{k}, j_{k}\right) \leq t\right\}\right)=\mathbb{P}\left(\cap_{k=1}^{l}\left\{h_{t}^{\eta}\left(i_{k}-j_{k}\right) \geq i_{k}+j_{k}\right\}\right) .
$$

From (2.7) we can also see that, in order to hole $i$ to interchange position with particle $j$, hole $i-1$ must have interchanged its position with particle $j$ and hole $i$ must have interchanged its position with particle $j-1$. After that, particle $j$ is placed at site $i-j-1$, waiting to interchange its position with hole $i$, that is placed at site $i-j$. Thus, for $i>U(j)$ we have that

$$
G^{\eta}(i, j)=w_{i, j}+\max \left\{G^{\eta}(i-1, j), G^{\eta}(i, j-1)\right\}_{+},
$$

where $w_{i, j}$ is the waiting time that particle $j$ jumps from site $i-j-1$ to site $i-j$. By the Markov property, the random variables $w_{i, j}$, for $j \in \mathbb{Z}$ and $i>U^{\eta}(j)$, are i.i.d. with an exponential distribution of parameter 1 .

Equation (2.9) enables us to see the interchange times $G$ as a optimisation problem related to oriented paths on $\mathbb{Z}^{2}$, called last-passage percolation model. The random environment in this setting is provided by a collection $\omega \equiv\left\{\omega_{\mathbf{z}}: \mathbf{z} \in \mathbb{Z}^{2}\right\}$ of i.i.d. random variables (passage times) with exponential distribution of parameter 1. For $\mathbf{x}, \mathbf{y} \in \mathbb{Z}^{2}$ with $\mathbf{x} \leq \mathbf{y}$ (coordinate-wise), we say that a sequence $\pi=\left(\pi_{0}, \pi_{1}, \cdots, \pi_{k}\right)$ is an up-right path from $\mathbf{x}$ to $\mathbf{y}$, if $\pi_{i+1}-\pi_{i} \in\{(1,0),(0,1)\}$, 
$\pi_{0}=\mathbf{x}$ and $\pi_{k}=\mathbf{y}$. Let $\Pi(\mathbf{x}, \mathbf{y})$ denote the collection of all up-right paths from $\mathbf{x}$ to $\mathbf{y}$. The last-passage percolation time from $\mathbf{x}$ to $\mathbf{y}$ is defined as

$$
L(\mathbf{x}, \mathbf{y}):=\max _{\pi \in \Pi(\mathbf{x}, \mathbf{y})} \sum_{i=0}^{k} \omega_{\pi_{i}}
$$

We do not have super-additivity for $L(\mathbf{x}, \mathbf{y})$, since for each path $\pi \in \Pi(\mathbf{x}, \mathbf{y})$ we include both end points in the sum of the passage times. This implies that, when concatenating paths, we add twice the passage time at the concatenation point. However, it holds that

$$
L(\mathbf{x}, \mathbf{y}) \geq L(\mathbf{x}, \mathbf{z})+L(\mathbf{z}, \mathbf{y})-\omega_{\mathbf{z}}
$$

for all $\mathbf{x} \leq \mathbf{z} \leq \mathbf{y}$. For convenience, we set $L(\mathbf{x}, \mathbf{y})=-\infty$ if $\mathbf{x} \leq \mathbf{y}$ does not hold. Given $\eta \in\{0,1\}^{\mathbb{Z}}$, recall (2.6) and set $\mathbf{x}_{j}^{\eta}:=\left(U^{\eta}(j)+1, j\right)$. For $j \in \mathbb{Z}$ and $i>U^{\eta}(j)$ define

$$
L^{\eta}(i, j)=\max _{k: \mathbf{x}_{k} \leq(i, j)} L\left(\mathbf{x}_{k},(i, j)\right) .
$$

For convenience, we also set $L^{\eta}(i, j)=-\infty$ if $i \leq U^{\eta}(j)$. The maximal path $\pi^{\eta}(i, j)$ is the a.s. unique up-right path $^{2}$, starting at some $\mathbf{x}_{k}$ such that $\mathbf{x}_{k} \leq(i, j)$, and ending at $(i, j)$ such that

$$
L^{\eta}(i, j)=\sum_{\mathbf{z} \in \pi^{\eta}(\mathbf{x})} \omega_{\mathbf{z}}
$$

For $i>0$ and $j>0$, consider the last point of $\pi^{\eta}(i, j)$ (in the up-right orientation) that intersects the positive coordinate axes $\{\mathbf{z}=(z, 0)$ or $\mathbf{z}=(0, z)$ and $z>0\}$. If such a point does not exist ${ }^{3}$ we must have that $\pi^{\eta}(i, j)$ starts at $(1,1)$, and in such a case we define $Z=1$. To distinguish between intersections via the horizontal- and vertical-axis we introduce a non-zero integer-valued random variable $Z=Z^{\eta}(i, j)$ such that

$$
L^{\eta}(\mathbf{x})= \begin{cases}L^{\eta}(Z, 0)+L((Z, 1), \mathbf{x}) & \text { if } Z>0 \\ L^{\eta}(0, Z)+L((1, Z), \mathbf{x}) & \text { if } Z<0 .\end{cases}
$$

To establish the connection with TASEP, notice that

$$
L^{\eta}(i, j)=\omega_{(i, j)}+\max \left\{L^{\eta}(i-1, j), L^{\eta}(i, j-1)\right\}_{+},
$$

where the similarity with (2.9) is evident. Hence,

$$
\left\{G^{\eta}(i, j): j \in \mathbb{Z} \text { and } i>U^{\eta}(j)\right\} \stackrel{\text { dist. }}{=}\left\{L^{\eta}(i, j): j \in \mathbb{Z} \text { and } i>U^{\eta}(j)\right\} \text {. }
$$

By (2.12), (2.8) and (2.5), the KPZ fixed point can be obtained as the limit fluctuations of $L^{\eta}$ as well. Denote

$$
[k]_{n} \equiv(n+k, n-k) \text { and } L^{\eta}[k]_{n} \equiv L^{\eta}(n+k, n-k) .
$$

For $x \in \mathbb{R}$ with $|x| \leq n$ we set $L^{\eta}[x]_{n}$ by linear interpolation of the values of $L^{\eta}[\cdot]_{n}$ at integer numbers. For a given sequence of initial profiles $\left(\eta^{(n)}, n \geq 1\right)$ define the process

$$
H_{t, n}(x) \equiv H_{t, n}\left(x ; \eta^{(n)}\right)=\frac{L^{\eta^{(n)}}\left[2^{2 / 3} x n^{2 / 3}\right]_{\lfloor t n\rfloor}-4 t n}{2^{4 / 3} n^{1 / 3}}
$$

\footnotetext{
${ }^{2}$ Almost sure uniqueness follows by continuity of the passage time distribution.

${ }^{3}$ Since $\mathbf{x}_{k}=\left(U^{\eta}(k)+1, k\right)$ and $U^{\eta}(k)=k+P(k) \leq k-1$ for all $k>1$, then $\pi^{\eta}(i, j)$ does not intersect the positive coordinate axes if and only if it starts at $\mathbf{x}_{1}$ and $U^{\eta}(1)=0$, which means that $\mathbf{x}_{1}=(1,1)$.
} 
if $\left|2^{2 / 3} x n^{2 / 3}\right| \leq n$, and set $H_{t, n}(x)=0$ otherwise. In view of (2.8), under (2.4), convergence of the finite dimensional distributions of $H_{t, n}$ to the finite dimensional distributions of $\mathfrak{h}_{t}$ follows from (2.5). It is also known that $\left\{H_{t, n}: n \geq 1\right\}$ is relatively compact (Corwin et al., 2016; Ferrari and Occelli, 2018; Pimentel, 2018) with respect to weak convergence of measures in $\mathrm{C}$ endowed with the uniform norm $\|\cdot\|_{\infty, a}$ on $[-a, a]$ (Billingsley, 1968). Thus, under (2.4),

$$
\lim _{n \rightarrow \infty} H_{t, n}\left(\cdot ; \eta^{(n)}\right) \stackrel{\text { dist. }}{=} \mathfrak{h}_{t}(\cdot ; \mathfrak{h})
$$

holds with respect to the uniform topology of local convergence in C.

\section{Last-Passage Percolation with Boundary Condition}

To apply the coupling method, it will be more convenient to work with a LPP model with boundary condition instead of having a down-right path as an initial profile. The boundary condition

$$
\mathrm{b}:=\left\{\omega_{\mathbf{x}}^{\mathrm{b}}: \text { for } \mathbf{x}=(z, 0) \text { or } \mathbf{x}=(0, z) \text { and } z \geq 0\right\},
$$

is given by real numbers $\omega_{\mathbf{x}}^{\mathrm{b}} \geq 0$ that are placed along the non-negative coordinate axes, and we will always assume that $\omega_{(0,0)}^{\mathrm{b}}=0$. Define

$$
\mathrm{b}(z)= \begin{cases}\sum_{i=1}^{-z} \omega_{0, i}^{\mathrm{b}} & \text { for } z<0 \\ 0 & \text { for } z=0 \\ \sum_{i=1}^{z} \omega_{i, 0}^{\mathrm{b}} & \text { for } z>0,\end{cases}
$$

and for $(k, l)>(0,0)$ and $z \in[-l, k]$,

$$
L_{z}(k, l)= \begin{cases}L((1,-z),(k, l)) & \text { for } z \in[-l, 0) \\ L((z, 1),(k, l)) & \text { for } z \in(0, k]\end{cases}
$$

The last-passage percolation time to $\mathbf{x}=(k, l)>(0,0)$, with boundary condition $\mathrm{b}$, is defined as

$$
\bar{L}^{\mathrm{b}}(k, l):=\max _{z \in[-l, k] \backslash\{0\}}\left\{\mathrm{b}(z)+L_{z}(k, l)\right\} .
$$

The exit point associated to the boundary condition $\mathrm{b}$ is defined as

$$
\bar{Z}^{\mathrm{b}}(k, l):=\max \arg \max _{z \in[-l, k] \backslash\{0\}}\left\{\mathrm{b}(z)+L_{z}(k, l)\right\},
$$

which corresponds to the right-most point $z \in[-l, k] \backslash\{0\}$ for which $\mathrm{b}(z)+L_{z}(k, l)=$ $\bar{L}^{\mathrm{b}}(k, l)$. Note that for fixed $n \geq 1$,

$$
\bar{Z}^{\mathrm{b}}(n+i, n-i) \text { is a non decreasing function of } i \in[-n, n] \text {. }
$$

Example 3.1 (Invariant regime). Let $\rho \in(0,1)$ and define a collection of independent random variables with $\omega_{(0,0)}^{\rho}=0$,

$$
\omega_{(z, 0)}^{\rho} \stackrel{\text { dist. }}{=} \operatorname{Exp}_{1}(1-\rho) \text { and } \omega_{(0, z)}^{\rho} \stackrel{\text { dist. }}{=} \operatorname{Exp}_{1}(\rho) \text { for } z \geq 1
$$

This boundary condition has a connection with the TASEP process started from $\operatorname{Bernoulli}(\rho)$ measure conditioned on $\eta(0)=0$ (hole at the origin) and $\eta(1)=1$ (particle at site one) (Balázs et al., 2006). A fundamental property that we will use is that the increments of the last-passage times $\bar{L}^{\rho}$ along the anti-diagonal are i.i.d. with a well known distribution. Precisely, define (recall that $[k]_{n}:=(n+k, n-k)$ )

$$
\zeta_{k, n}^{\rho}:=\bar{L}^{\rho}[k]_{n}-\bar{L}^{\rho}[k-1]_{n}, \text { for } k=-n+1, \ldots, n .
$$


Then $\left\{\zeta_{k, n}^{\rho}: k=-n+1, \ldots, n\right\}$ is a collection of independent random variables with

$$
\zeta_{k, n}^{\rho} \stackrel{\text { dist. }}{=} \operatorname{Exp}_{1}(1-\rho)-\operatorname{Exp}_{2}(\rho)
$$

where $\operatorname{Exp}_{1}(1-\rho)$ and $\operatorname{Exp}_{2}(\rho)$ are independent random variables with exponential distribution of parameter $\rho$ and $1-\rho$, respectively (see Lemma 4.2 in Balázs et al., 2006).

Example 3.2 (Boundary induced by curve to point LPP). Let $\omega^{\eta}(0,0)=0$, and for $z \geq 1$ set

$$
\omega^{\eta}(z, 0):=L^{\eta}(0, z)-L^{\eta}(z-1,0) \text { and } \omega^{\eta}(0, z):=L^{\eta}(0, z)-L^{\eta}(0, z-1) .
$$

(Notice that $L^{\eta}(0,0)=0$ since $(0,0) \in \Gamma$.) Then

$$
\bar{L}^{\eta}(k, l)=L^{\eta}(k, l) \text { and } \bar{Z}^{\eta}(k, l)=Z^{\eta}(k, l) \text {, for all } k, l>0 .
$$

To see this, first consider $z \in(0, k]$. Then,

$$
\mathrm{b}^{\eta}(z)+L_{z}(k, l)=L^{\eta}(0, z)+L_{z}(k, l) \leq L^{\eta}(k, l) .
$$

Similarly we have the same inequality for $z \in[-l, 0)$, and hence $\bar{L}^{\eta}(k, l) \leq L^{\eta}(k, l)$. On the other hand, if one take $Z=Z^{\eta}(k, l)$ (as defined at the end of Section 2) then

$$
L^{\eta}(k, l)=\mathrm{b}^{\eta}(Z)+L_{Z}(k, l) \leq \bar{L}^{\eta}(k, l) .
$$

and thus $L^{\eta}(k, l) \leq \bar{L}^{\eta}(k, l)$.

3.1. Argmax Comparison and Attractiveness under the Basic Coupling. Given b and $\tilde{\mathrm{b}}$, the basic coupling is the joint construction of $\left(L^{\mathrm{b}}, L^{\tilde{\mathrm{b}}}\right)$ using the same pointto-point last-passage percolation times (3.1):

$$
\begin{aligned}
& \bar{L}^{\mathrm{b}}(k, l):=\max _{z \in[-l, k] \backslash\{0\}}\left\{\mathrm{b}(z)+L_{z}(k, l)\right\} \\
& \text { and } \bar{L}^{\tilde{\mathrm{b}}}(k, l):=\max _{z \in[-l, k] \backslash\{0\}}\left\{\tilde{\mathrm{b}}(z)+L_{z}(k, l)\right\} .
\end{aligned}
$$

The next lemma allow us to compare, under the basic coupling, local increments associated to different boundary conditions by looking at the relative positions of the respective exit points.

Lemma 3.3 (Argmax Comparison). Under (3.7),

$$
\text { if } \bar{Z}^{\mathrm{b}}[j]_{n} \leq \bar{Z}^{\tilde{\mathrm{b}}}[i]_{n} \text {, for } i \leq j \text {, then } \bar{L}^{\mathrm{b}}[j]_{n}-\bar{L}^{\mathrm{b}}[i]_{n} \leq \bar{L}^{\tilde{\mathrm{b}}}[j]_{n}-\bar{L}^{\tilde{\mathrm{b}}}[i]_{n} \text {. }
$$

Proof: This lemma is similar to Lemma 2.1 in Pimentel (2018), and the proof follows the same lines. Denote $\pi_{z}(\mathbf{x})$ the maximal path associated to $L_{z}(\mathbf{x})$. Denote $z_{1} \equiv \bar{Z}^{\mathrm{b}}[j]_{n}$ and $z_{2} \equiv \bar{Z}^{\tilde{\mathrm{b}}}[i]_{n}$. Let $\mathbf{c}$ be a crossing between $\pi_{z_{1}}\left([j]_{n}\right)$ and $\pi_{z_{2}}\left([i]_{n}\right)$. Such a crossing always exists because, by assumption, $i \leq j$ and $z_{1} \leq z_{2}$. We note that (2.10) still holds when boundaries are introduced, and hence

$$
\bar{L}^{\tilde{\mathrm{b}}}[j]_{n} \geq \tilde{\mathrm{b}}\left(z_{2}\right)+L_{z_{2}}\left([j]_{n}\right) \geq \tilde{\mathrm{b}}\left(z_{2}\right)+L_{z_{2}}(\mathbf{c})+L\left(\mathbf{c},[j]_{n}\right)-\omega_{\mathbf{c}} .
$$

We use this, and that (since $\mathbf{c} \in \pi_{z_{2}}\left([i]_{n}\right)$ )

$$
\tilde{\mathrm{b}}\left(z_{2}\right)+L_{z_{2}}(\mathbf{c})-\bar{L}^{\tilde{\mathrm{b}}}[i]_{n}=-L\left(\mathbf{c},[i]_{n}\right)+\omega_{\mathbf{c}}
$$


in the following inequality:

$$
\begin{aligned}
\bar{L}^{\tilde{\mathrm{b}}}[j]_{n}-\bar{L}^{\tilde{\mathrm{b}}}[i]_{n} & \geq \tilde{\mathrm{b}}\left(z_{2}\right)+L_{z_{2}}(\mathbf{c})+L\left(\mathbf{c},[j]_{n}\right)-\omega_{\mathbf{c}}-L^{\tilde{\mathrm{b}}}[i]_{n} \\
& =L\left(\mathbf{c},[j]_{n}\right)-L\left(\mathbf{c},[i]_{n}\right) .
\end{aligned}
$$

By (2.10),

$$
-L\left(\mathbf{c},[i]_{n}\right) \geq \bar{L}^{\mathrm{b}}(\mathbf{c})-\bar{L}^{\mathrm{b}}[i]_{n}-\omega_{\mathbf{c}}
$$

and hence (since $\mathbf{c} \in \pi_{z_{1}}\left([j]_{n}\right)$ )

$$
\begin{aligned}
\bar{L}^{\tilde{\mathrm{b}}}[j]_{n}-\bar{L}^{\tilde{\mathrm{b}}}[i]_{n} & \geq L\left(\mathbf{c},[j]_{n}\right)-L\left(\mathbf{c},[i]_{n}\right) \\
& \geq L\left(\mathbf{c},[j]_{n}\right)+\bar{L}^{\mathrm{b}}(\mathbf{c})-L^{\mathrm{b}}[i]_{n}-\omega_{\mathbf{c}} \\
& =\bar{L}^{\mathrm{b}}(\mathbf{c})+\left(L\left(\mathbf{c},[j]_{n}\right)-\omega_{\mathbf{c}}\right)-L^{\mathrm{b}}[i]_{n} \\
& =\bar{L}^{\mathrm{b}}[j]_{n}-\bar{L}^{\mathrm{b}}[i]_{n} .
\end{aligned}
$$

Another useful property is attractiveness of the LPP model with boundary condition. It states that, under the basic coupling, if one starts the with ordered boundary conditions then the last-passage percolation times remain ordered as well.

Lemma 3.4 (Attractiveness). Under (3.7), if $\mathrm{b}(j)-\mathrm{b}(i) \leq \tilde{\mathrm{b}}(j)-\tilde{\mathrm{b}}(i)$, for all $i \leq j$, then

$$
\bar{L}^{\mathrm{b}}[j]_{n}-\bar{L}^{\mathrm{b}}[i]_{n} \leq \bar{L}^{\tilde{\mathrm{b}}}[j]_{n}-\bar{L}^{\tilde{\mathrm{b}}}[i]_{n}, \forall i \leq j .
$$

Proof: Denote again $z_{1} \equiv Z^{\mathrm{b}}[j]_{n}$ and $z_{2}:=Z^{\tilde{\mathrm{b}}}[i]_{n}$. If $z_{1} \leq z_{2}$ then it follows from Lemma 3.3 (we do not need to use the assumption). If $z_{1}>z_{2}$ then

$$
\begin{aligned}
\bar{L}^{\tilde{\mathrm{b}}}[j]_{n}-\bar{L}^{\tilde{\mathrm{b}}}[i]_{n} & -\left(\bar{L}^{\mathrm{b}}[j]_{n}-\bar{L}^{\mathrm{b}}[i]_{n}\right) \\
& =\bar{L}^{\tilde{\mathrm{b}}}[j]_{n}-\left(\tilde{\mathrm{b}}\left(z_{2}\right)+L_{z_{2}}[i]_{n}\right)-\left(\left(\mathrm{b}\left(z_{1}\right)+L_{z_{1}}[j]_{n}\right)-\bar{L}^{\mathrm{b}}[i]_{n}\right) \\
& =\bar{L}^{\tilde{\mathrm{b}}}[j]_{n}-\left(\tilde{\mathrm{b}}\left(z_{2}\right)+L_{z_{1}}[j]_{n}\right)-\left(\left(\mathrm{b}\left(z_{1}\right)+L_{z_{2}}[i]_{n}\right)-\bar{L}^{\mathrm{b}}[i]_{n}\right) \\
& =\bar{L}^{\tilde{\mathrm{b}}}[j]_{n}-\left(\tilde{\mathrm{b}}\left(z_{2}\right)+L_{z_{1}}[j]_{n}\right)+\left(\bar{L}^{\mathrm{b}}[i]_{n}-\left(\mathrm{b}\left(z_{1}\right)+L_{z_{2}}[i]_{n}\right)\right) \\
& =\bar{L}^{\tilde{\mathrm{b}}}[j]_{n}-\left(\tilde{\mathrm{b}}\left(z_{1}\right)+L_{z_{1}}[j]_{n}\right)+\left(\bar{L}^{\mathrm{b}}[i]_{n}-\left(\mathrm{b}\left(z_{2}\right)+L_{z_{2}}[i]_{n}\right)\right) \\
& +\left(\tilde{\mathrm{b}}\left(z_{1}\right)-\tilde{\mathrm{b}}\left(z_{2}\right)\right)-\left(\mathrm{b}\left(z_{1}\right)-\mathrm{b}\left(z_{2}\right)\right) .
\end{aligned}
$$

On the other hand,

$$
\bar{L}^{\tilde{\mathrm{b}}}[j]_{n}-\left(\tilde{\mathrm{b}}\left(z_{1}\right)+L_{z_{1}}[j]_{n}\right) \geq 0
$$

and

while, by assumption,

$$
\bar{L}^{\mathrm{b}}[i]_{n}-\left(\mathrm{b}\left(z_{2}\right)+L_{z_{2}}[i]_{n}\right) \geq 0
$$

$$
\tilde{\mathrm{b}}\left(z_{1}\right)-\tilde{\mathrm{b}}\left(z_{2}\right) \geq \mathrm{b}\left(z_{1}\right)-\mathrm{b}\left(z_{2}\right)
$$

since $z_{1}>z_{2}$.

Lemma 3.5. Under (3.7), if $\mathrm{b}(j)-\mathrm{b}(i) \leq \tilde{\mathrm{b}}(j)-\tilde{\mathrm{b}}(i)$, for all $i \leq j$, then $0 \leq\left(\bar{L}^{\tilde{\mathrm{b}}}[i]_{n}-\bar{L}^{\tilde{\mathrm{b}}}[0]_{n}\right)-\left(\bar{L}^{\mathrm{b}}[i]_{n}-\bar{L}^{\mathrm{b}}[0]_{n}\right) \leq\left(\bar{L}^{\tilde{\mathrm{b}}}[j]_{n}-\bar{L}^{\tilde{\mathrm{b}}}[0]_{n}\right)-\left(\bar{L}^{\mathrm{b}}[j]_{n}-\bar{L}^{\mathrm{b}}[0]_{n}\right)$, 
for all $0 \leq i \leq j$, and

$0 \leq\left(\bar{L}^{\mathrm{b}}[i]_{n}-\bar{L}^{\mathrm{b}}[0]_{n}\right)-\left(\bar{L}^{\tilde{\mathrm{b}}}[i]_{n}-\bar{L}^{\tilde{\mathrm{b}}}[0]_{n}\right) \leq\left(\bar{L}^{\mathrm{b}}[j]_{n}-\bar{L}^{\mathrm{b}}[0]_{n}\right)-\left(\bar{L}^{\tilde{\mathrm{b}}}[j]_{n}-\bar{L}^{\tilde{\mathrm{b}}}[0]_{n}\right)$, for all $j \leq i \leq 0$.

Proof: It follows from Lemma 3.4 since the first inequality is equivalent to

$$
\bar{L}^{\mathrm{b}}[j]_{n}-\bar{L}^{\mathrm{b}}[i]_{n} \leq \bar{L}^{\tilde{\mathrm{b}}}[j]_{n}-\bar{L}^{\tilde{\mathrm{b}}}[i]_{n},
$$

for $i \leq j$, while the second one is equivalent to

$$
\bar{L}^{\mathrm{b}}[i]_{n}-\bar{L}^{\mathrm{b}}[j]_{n} \leq \bar{L}^{\tilde{\mathrm{b}}}[i]_{n}-\bar{L}^{\tilde{\mathrm{b}}}[j]_{n},
$$

for $j \leq i$. Notice that $i \geq 0$ implies that

$$
0 \leq\left(\bar{L}^{\tilde{\mathrm{b}}}[i]_{n}-\bar{L}^{\tilde{\mathrm{b}}}[0]_{n}\right)-\left(\bar{L}^{\mathrm{b}}[i]_{n}-\bar{L}^{\mathrm{b}}[0]_{n}\right),
$$

and $i \leq 0$ implies that

$$
0 \leq\left(\bar{L}^{\mathrm{b}}[i]_{n}-\bar{L}^{\tilde{\mathrm{b}}}[0]_{n}\right)-\left(\bar{L}^{\tilde{\mathrm{b}}}[i]_{n}-\bar{L}^{\tilde{\mathrm{b}}}[0]_{n}\right)
$$

3.2. KPZ Localisation of Exit Points. For $x \in \mathbb{R}$ and $t \geq 0$, we denote

$$
[x]_{t} \equiv(\lfloor t\rfloor+\lfloor x\rfloor,\lfloor t\rfloor-\lfloor x\rfloor) .
$$

Recall the definition (2.11) of $Z^{\eta}$, denote $\mathbf{y}_{t, n}^{ \pm}:=\left[ \pm(2 t n)^{2 / 3}\right]_{t n}$ and let $\phi_{t}(r):=\underset{n}{\limsup } \mathbb{P}\left(\left|Z^{\eta^{(n)}}\left(\mathbf{y}_{t, n}^{+}\right)\right|>r(t n)^{2 / 3}\right)+\underset{n}{\limsup } \mathbb{P}\left(\left|Z^{\eta^{(n)}}\left(\mathbf{y}_{t, n}^{-}\right)\right|>r(t n)^{2 / 3}\right)$.

The proof of the following proposition is postponed to Section 5.

Proposition 3.6. Under assumption (1.6), there exist $t_{0}>0$ and $\phi(r)$ such that $\phi_{t}(r) \leq \phi(r)$ for all $t \geq t_{0}$ and $\lim _{r \rightarrow \infty} \phi(r)=0$.

In the stationary LPP model with boundary, the macroscopic location of the exit point $Z^{\rho}(n, n)$ is $\left(1-d_{\rho}\right) n$, where

$$
d_{\rho}=\left(\frac{1-\rho}{\rho}\right)^{2} .
$$

For $\rho=1 / 2$ we have $d_{\rho}=1$ and the exit point stays close to the origin with fluctuations of order $n^{2 / 3}$ (Balázs et al., 2006). If one introduces a small perturbation of density $1 / 2$ by setting $\rho_{n}^{ \pm}=1 / 2 \pm c r n^{-1 / 3}$ then $Z^{\rho_{x}^{ \pm} n}(n, n)$ fluctuates around $\left(1-d_{\rho}\right) n \sim \pm r n^{2 / 3}$, which allows us to tune $\rho$ in such way that, with high probability, $Z^{\rho^{-}}(n, n) \leq-\epsilon r n^{2 / 3} \leq \epsilon r n^{2 / 3} \leq Z^{\rho^{+}}(n, n)$ for some small $\epsilon>0$ that only depends on $c$.

Lemma 3.7. Set $\rho_{n}^{ \pm}:=\frac{1}{2} \pm c \frac{r}{n^{1 / 3}}$, where $c>0$ is fixed. There exist constants $\epsilon_{1}, C_{1}>0$ such that, for all $r>1$,

$$
\limsup _{n \rightarrow \infty} \mathbb{P}\left(\bar{Z}^{\rho_{n}^{+}}\left[-(2 n)^{2 / 3}\right]_{n}<\epsilon_{1} r n^{2 / 3}\right) \leq \frac{C_{1}}{r^{3}},
$$

and

$$
\limsup _{n \rightarrow \infty} \mathbb{P}\left(\bar{Z}^{\rho_{n}^{-}}\left[(2 n)^{2 / 3}\right]_{n}>-\epsilon_{1} r n^{2 / 3}\right) \leq \frac{C_{1}}{r^{3}}
$$


Proof: The proof of this lemma follows the same argument in the proof of Lemma 2.3 of Pimentel (2018), that is based on the fluctuations results for exitpoints provided by Balázs et al. (2006). More recently, this upper bound was improved in Seppäläinen and Shen (2020) to $\mathrm{Ce}^{-c r^{3}}$ (see Theorem 3.5 and Lemma 4.2 there). At a first glance this lemma could appear counter intuitive since for fixed $\rho$ the probabilities are increasing with $r$ while the upper bound is decreasing with $r$. However, one should notice that the parameter $\rho$ does depend on $r$ (and $n$ ). In the $n \rightarrow \infty$ limit, the corresponding initial height profile is a Brownian motion with drift $\mu^{ \pm}= \pm a r$, where $a>0$ is a constant depending on $c$ (Baik et al., 2010; Corwin et al., 2016). By (3.2) the exit point converges under KPZ scaling to

$$
Z^{\mu^{ \pm}}[x] \stackrel{\text { dist. }}{=} \underset{z}{\arg \max }\left\{\mathfrak{b}(z)+\mu_{ \pm} z+\mathcal{A}(z)-(z-x)^{2}\right\} .
$$

Notice that

$$
\mu_{ \pm} z-(z-x)^{2}=-\left(z-\left(x+\frac{\mu_{ \pm}}{2}\right)\right)^{2}+\frac{\mu_{ \pm}^{2}}{4}+\mu_{ \pm} x
$$

and the vertical shift $\left(\mu_{ \pm}^{2} / 4+\mu_{ \pm} x\right)$ the does not affect the location of the maximum (recall the Affine Symmetry (ii)). Thus,

$$
Z^{\mu^{ \pm}}[x] \stackrel{\text { dist. }}{\arg \max }\left\{\mathfrak{z}(z)+\mathcal{A}(z)-(z-u)^{2}\right\} \stackrel{\text { dist. }}{=} Z^{0}[0]+u,
$$

where $u=x+\mu_{ \pm} / 2$. Therefore,

$$
\mathbb{P}\left(Z^{\mu}[1]<\epsilon r\right)=\mathbb{P}\left(Z^{0}[0]<\epsilon r-\frac{\mu}{2}-1\right)=\mathbb{P}\left(Z^{0}[0]<\left(\epsilon-\frac{a}{2}\right) r-1\right),
$$

and if we choose $\epsilon=a / 4$ we see that

$$
\mathbb{P}\left(Z^{\mu}[1]<\epsilon r\right)=\mathbb{P}\left(Z^{0}[0]<-\frac{a}{4} r-1\right) \rightarrow 0 \text { as } r \rightarrow \infty .
$$

This brief explanation sheds some light in rule of $\epsilon_{1}$, that appears to take in to account the presence of the drift in $\rho_{n}^{ \pm}$. To prove the first upper bound, one can use that (see Figure 4.2 and Lemma 4.3 in Seppäläinen and Shen, 2020)

$$
\mathbb{P}\left(\bar{Z}^{\rho}(k, l)<i\right)=\mathbb{P}\left(\bar{Z}^{\rho}(k-i, l+j)<-j\right) .
$$

Thus, for $k_{n}=n-\left\lfloor(2 n)^{2 / 3}\right\rfloor, l_{n}=n+\left\lfloor(2 n)^{2 / 3}\right\rfloor$ and $i_{n}=\left\lfloor\epsilon_{1} r n^{2 / 3}\right\rfloor+1$, we get

$$
\mathbb{P}\left(\bar{Z}^{\rho_{n}^{+}}\left[-(2 n)^{2 / 3}\right]_{n}<\epsilon_{1} r n^{2 / 3}\right) \leq \mathbb{P}\left(\bar{Z}^{\rho_{n}^{+}}\left(k_{n}-i_{n}, l_{n}+j\right)<-j\right),
$$

for all $j \in \mathbb{Z}_{+}$. Now, we choose $j_{n}$ such that

$$
\left\{\begin{array}{l}
k_{n}-i_{n}=m_{n} \\
l_{n}+j_{n}=\left\lfloor d_{\rho}^{-1} m_{n}\right\rfloor .
\end{array}\right.
$$

From Balázs et al. (2006), we know that there exists $m_{0} \geq 1$ such that

$$
\mathbb{P}\left(\left|Z^{\rho}\left(m,\left\lfloor d_{\rho}^{-1} m\right\rfloor\right)\right| \geq r m^{2 / 3}\right) \leq \frac{C}{r^{3}},
$$

for all $m \geq m_{0}$ and $r>1$, where the constant $C$ depends only on $n_{0}$ and $\rho$, which can be uniformly bounded for all $\rho \in[a, b] \subseteq(0,1)$. In our case $\rho=\rho_{n}^{+} \in[1 / 4,3 / 4]$ for large enough $n$, and to complete the proof it suffices to verify that $j_{n}$ is bounded below by $\bar{\epsilon} r m_{n}^{2 / 3}$ for a suitable $\epsilon_{1}>0$. 
By (3.6) we have that,

$$
\phi_{t}(r)=\underset{n}{\limsup } \mathbb{P}\left(\left|\bar{Z}^{\eta^{(n)}}\left(\mathbf{y}_{t, n}^{+}\right)\right|>r(t n)^{2 / 3}\right)+\underset{n}{\limsup } \mathbb{P}\left(\left|\bar{Z}^{\eta^{(n)}}\left(\mathbf{y}_{t, n}^{-}\right)\right|>r(t n)^{2 / 3}\right) \text {. }
$$

Lemma 3.8. For $r>0$ let

$$
\rho_{t, n}^{ \pm} \equiv \rho_{t, n}^{ \pm}(r):=\frac{1}{2} \pm c \frac{r}{\lfloor n\rfloor^{1 / 3}},
$$

and

$$
E_{t, n}^{\eta^{(n)}}(r):=\left\{\bar{Z}^{\rho_{t, n}^{+}}\left(\mathbf{y}_{t, n}^{-}\right) \geq \bar{Z}^{\eta^{(n)}}\left(\mathbf{y}_{t, n}^{+}\right) \text {and } \bar{Z}^{\rho_{t, n}^{-}}\left(\mathbf{y}_{t, n}^{+}\right) \leq \bar{Z}^{\eta^{(n)}}\left(\mathbf{y}_{t, n}^{-}\right)\right\}
$$

Then,

$$
\limsup _{n \rightarrow \infty} \mathbb{P}\left(E_{t, n}^{\eta^{(n)}}(r)^{c}\right) \leq 2 C_{1} r^{-3}+\phi_{t}\left(2^{-1} \epsilon_{1} r\right)
$$

where $\epsilon_{1}$ and $C_{1}$ are given by Lemma 3.7.

Proof: The probability of $E_{t, n}^{\eta^{(n)}}(r)^{c}$ is bounded above by

$$
\mathbb{P}\left(\bar{Z}^{\rho_{t, n}^{+}}\left(\mathbf{y}_{t, n}^{-}\right)<\bar{Z}^{\eta^{(n)}}\left(\mathbf{y}_{t, n}^{+}\right)\right)+\mathbb{P}\left(\bar{Z}^{\rho_{t, n}^{-}}\left(\mathbf{y}_{t, n}^{+}\right)>\bar{Z}^{\eta^{(n)}}\left(\mathbf{y}_{t, n}^{-}\right)\right) .
$$

To deal with the first term in (3.9), we note that

$$
\begin{aligned}
\mathbb{P}\left(\bar{Z}^{\rho_{t, n}^{+}}\left(\mathbf{y}_{t, n}^{-}\right)<\bar{Z}^{\eta^{(n)}}\left(\mathbf{y}_{t, n}^{+}\right)\right) & \leq \mathbb{P}\left(\left|\bar{Z}^{\eta^{(n)}}\left(\mathbf{y}_{t, n}^{+}\right)\right|>2^{-1} \epsilon_{1} r(t n)^{2 / 3}\right) \\
& +\mathbb{P}\left(\bar{Z}^{\rho_{t, n}^{+}}\left(\mathbf{y}_{t, n}^{-}\right)<2^{-1} \epsilon_{1} r(t n)^{2 / 3}\right) .
\end{aligned}
$$

Set $m=\lfloor t n\rfloor$ and using Lemma $3.7\left(2^{-1}(n t)^{2 / 3} \leq\lfloor n t\rfloor^{2 / 3}\right.$ for large $\left.n\right)$

$$
\begin{aligned}
& \limsup _{n \rightarrow \infty} \mathbb{P}\left(\bar{Z}^{\rho_{t, n}^{+}}\left(\mathbf{y}_{t, n}^{-}\right)<2^{-1} \epsilon_{1} r(t n)^{2 / 3}\right) \\
& \quad \leq \limsup _{m \rightarrow \infty} \mathbb{P}\left(\bar{Z}^{\rho_{m}^{+}}\left[-(2 m)^{2 / 3}\right]_{m}<\epsilon_{1} r m^{2 / 3}\right) .
\end{aligned}
$$

The analogous upper bound for the second term in (3.9) is obtained by using that

$$
\begin{aligned}
\mathbb{P}\left(\bar{Z}^{\rho_{t, n}^{-}}\left(\mathbf{y}_{t, n}^{+}\right)>\bar{Z}^{\eta^{(n)}}\left(\mathbf{y}_{t, n}^{-}\right)\right) & \leq \mathbb{P}\left(\left|\bar{Z}^{\eta^{(n)}}\left(\mathbf{y}_{t, n}^{-}\right)\right|>2^{-1} \epsilon_{1} r(t n)^{2 / 3}\right) \\
& +\mathbb{P}\left(\bar{Z}^{\rho_{t, n}^{-}}\left(\mathbf{y}_{t, n}^{+}\right)>-2^{-1} \epsilon_{1} r(t n)^{2 / 3}\right) .
\end{aligned}
$$

\section{Proof of the Theorem 1.1}

Now we are ready to construct the coupling $\left(\mathfrak{h}_{t}(\cdot ; \mathfrak{h}), \mathfrak{h}_{t}(\cdot ; \mathfrak{b})\right)$ and get the upper bound (1.7). Given $\mathfrak{h} \in U$ UC, we pick a sequence of particle configurations $\eta^{(n)}$ such that (2.4) holds. Recall that for $x \in \mathbb{R}$ and $|x| \leq n$, the last-passage percolation times $L^{\eta}[x]_{n}$ are defined by linear interpolation of the values at the integer numbers, so that

$$
H_{t, n}(x) \equiv H_{t, n}\left(x ; \eta^{(n)}\right)=\frac{L^{\eta^{(n)}}\left[2^{2 / 3} x n^{2 / 3}\right]_{\lfloor t n\rfloor}-4 n t}{2^{4 / 3} n^{1 / 3}},
$$

where $\left|2^{2 / 3} x n^{2 / 3}\right| \leq n$ and $H_{t, n}(x)=0$ otherwise, defines a collection of continuous random processes $\left\{H_{t, n}(\cdot): n \geq 1\right\}$. For fixed $t>0$, this collection is relatively 
compact (Corwin et al., 2016; Ferrari and Occelli, 2018; Pimentel, 2018) with respect to weak convergence of measures in C, and by (2.13)

$$
\lim _{n \rightarrow \infty} H_{t, n}(\cdot) \stackrel{\text { dist. }}{=} \mathfrak{h}_{t}(\cdot ; \mathfrak{h}) \text {. }
$$

For the two-sided Brownian motion $\mathfrak{b}$ we pick a particle configuration $\eta^{1 / 2}$ given by i.i.d. Bernoulli of parameter $1 / 2$, and set $H_{t, n}^{1 / 2}(\cdot) \equiv H_{t, n}\left(\cdot ; \eta^{1 / 2}\right)$. Thus, by (2.13),

$$
\lim _{n \rightarrow \infty} H_{t, n}^{1 / 2}(\cdot) \stackrel{\text { dist. }}{=} \mathfrak{h}_{t}(x ; \mathfrak{b}) \text {. }
$$

We use the same environment $\omega$ of i.i.d. exponential random variables of parameter 1 to couple $H_{t, n}$ and $H_{t, n}^{1 / 2}$. Since the sequences $\left(H_{t, n}\right)_{n \geq 1}$ and $\left(H_{t, n}^{1 / 2}\right)_{n \geq 1}$ are tight, we have that $\left(H_{t, n}, H_{t, n}^{1 / 2}\right)_{n \geq 1}$ is tight in $\mathrm{C} \times \mathrm{C}$ (endowed with the product topology). Therefore, $\left(H_{t, n}, H_{t, n}^{1 / 2}\right)_{n \geq 1}$ is relatively compact in $\mathrm{C} \times \mathrm{C}$ which implies the existence of a weak limit $\left(\mathfrak{h}_{t}(\cdot ; \mathfrak{h}), \mathfrak{h}_{t}(\cdot ; \mathfrak{b})\right)$ in $\mathbf{C} \times \mathbf{C}$, whose marginals are obviously given by $(1.1)$. We will show next that such a coupling $\left(\mathfrak{h}_{t}(\cdot ; \mathfrak{h}), \mathfrak{h}_{t}(\cdot ; \mathfrak{b})\right)$ satisfies (1.7).

Set $\delta_{t}:=a t^{-2 / 3}, r=r_{t}:=\delta_{t}^{-1 / 4}$ and $\rho_{n, t}^{ \pm}:=\rho_{n, t}^{ \pm}\left(r_{t}\right)$ (the value of $c>0$ will be given later). Given a profile $\omega_{\mathbf{x}}^{1 / 2}$ as in Example 3.1, define the boundary conditions $\mathrm{b}^{ \pm}$by setting

$$
\omega_{\mathbf{x}}^{ \pm}= \begin{cases}\frac{1}{2 \rho_{t, n}^{ \pm}} \omega_{(0,|z|)}^{1 / 2} & \text { for } z<0 \\ \frac{1}{2\left(1-\rho_{t, n}^{ \pm}\right)} \omega_{(z, 0)}^{1 / 2} & \text { for } z>0 .\end{cases}
$$

Thus, $\omega^{ \pm} \stackrel{\text { dist. }}{=} \omega^{\rho_{n, t}^{ \pm}}$. Since $\rho_{n, t}^{-} \leq 1 / 2 \leq \rho_{t, n}^{+}$, if $i<0$ then,

$$
\omega_{(0,-i)}^{-}-\omega_{(0,-i)}^{1 / 2}=\left(\frac{1}{2 \rho_{n, t}^{-}}-1\right) \omega_{(0,-i)}^{1 / 2} \geq 0,
$$

and

$$
\omega_{(0,-i)}^{+}-\omega_{(0,-i)}^{1 / 2}=\left(\frac{1}{2 \rho_{n, t}^{+}}-1\right) \omega_{(0,-i)}^{1 / 2} \leq 0
$$

If $i>0$,

$$
\omega_{(i, 0)}^{-}-\omega_{(i, 0)}^{1 / 2}=\left(\frac{1}{2\left(1-\rho_{n, t}^{-}\right)}-1\right) \omega_{(0,-i)}^{1 / 2} \leq 0
$$

and

$$
\omega_{(i, 0)}^{+}-\omega_{(i, 0)}^{1 / 2}=\left(1-\frac{1}{2\left(1-\rho_{n, t}^{+}\right)}\right) \omega_{(i, 0)}^{(1 / 2)}, \geq 0 .
$$

Hence, if $i<j$ then

$$
\mathrm{b}^{-}(j)-\mathrm{b}^{-}(i) \leq \mathrm{b}^{+}(j)-\mathrm{b}^{+}(i) .
$$

Again, in order to simplify the notation, we use the superscript \pm for quantities that are related to $\rho_{n, t}^{ \pm}$, and the last-passages times $\bar{L}^{ \pm} \equiv \bar{L}^{\rho_{n, t}^{ \pm}}, \bar{L}^{\eta^{(n)}}=L^{\eta^{(n)}}$ and $\bar{L}^{\eta^{1 / 2}}=L^{\eta^{1 / 2}}$ (recall Example 3.1 and Example 3.2) using the basic coupling (3.7). By (4.1) and Lemma 3.5, for $x \in[0, a]$,

$$
0 \leq \Delta \bar{H}_{t, n}^{+}(x)-\Delta \bar{H}_{t, n}^{-}(x) \leq \Delta \bar{H}_{t, n}^{+}(a)-\Delta \bar{H}_{t, n}^{-}(a),
$$


while for $x \in[-a, 0]$,

$$
0 \leq \Delta \bar{H}_{t, n}^{-}(x)-\Delta \bar{H}_{t, n}^{+}(x) \leq \Delta \bar{H}_{t, n}^{-}(-a)-\Delta \bar{H}_{t, n}^{+}(-a) .
$$

Recall Lemma 3.8 and let $E_{t, n} \equiv E_{t, n}^{\eta^{(n)}}\left(r_{t}\right)$ and $E_{t, n}^{1 / 2} \equiv E_{t, n}^{\eta^{1 / 2}}\left(r_{t}\right)$. Since $\delta_{t}:=$ $a t^{-2 / 3}$, then $a n^{2 / 3}=\delta_{t}(t n)^{2 / 3}$ and $\delta_{t} \leq 1$ for $t \geq a^{3 / 2}$. Thus,

$$
2^{2 / 3} a n^{2 / 3} \leq(2 t n)^{2 / 3} \text { and }-2^{2 / 3} a n^{2 / 3} \geq-(2 t n)^{2 / 3} .
$$

By (3.3), this implies that, on the event $E_{t, n}$,

$$
\bar{Z}^{+}\left[-2^{2 / 3} a n^{2 / 3}\right]_{t n} \geq \bar{Z}^{\eta^{(n)}}\left[2^{2 / 3} a n^{2 / 3}\right]_{t n}
$$

and

and on the event $E_{t, n}^{1 / 2}$,

$$
\bar{Z}^{-}\left[2^{2 / 3} a n^{2 / 3}\right]_{t n} \leq \bar{Z}^{\eta^{(n)}}\left[-2^{2 / 3} a n^{2 / 3}\right]_{t n},
$$

$$
\bar{Z}^{+}\left[-2^{2 / 3} a n^{2 / 3}\right]_{t n} \geq \bar{Z}^{\eta^{1 / 2}}\left[2^{2 / 3} a n^{2 / 3}\right]_{t n}
$$

and

$$
\bar{Z}^{-}\left[2^{2 / 3} a n^{2 / 3}\right]_{t n} \leq \bar{Z}^{\eta^{1 / 2}}\left[-2^{2 / 3} a n^{2 / 3}\right]_{t n} .
$$

By (3.3), on the event $E_{t, n}$, if $x \in[0, a]$ then

$$
\bar{Z}^{\eta^{(n)}}\left[2^{2 / 3} x n^{2 / 3}\right]_{t n} \leq \bar{Z}^{+}[0]_{t n} \text { and } \bar{Z}^{-}\left[2^{2 / 3} x n^{2 / 3}\right]_{t n} \leq \bar{Z}^{\eta^{(n)}}[0]_{t n},
$$

while if $x \in[-a, 0]$ then

$$
\bar{Z}^{\eta^{(n)}}[0]_{t n} \leq \bar{Z}^{+}\left[2^{2 / 3} x n^{2 / 3}\right]_{t n} \text { and } \bar{Z}^{-}[0]_{t n} \leq \bar{Z}^{\eta^{(n)}}\left[2^{2 / 3} x n^{2 / 3}\right]_{t n} \text {. }
$$

By Lemma 3.3, for $x \in[0, a]$, (recall (3.6))

$$
\begin{aligned}
\bar{L}^{-}\left[2^{2 / 3} x n^{2 / 3}\right]_{t n}-\bar{L}^{-}[0]_{t n} & \leq L^{\eta^{(n)}}\left[2^{2 / 3} x n^{2 / 3}\right]_{t n}-L^{\eta^{(n)}}[0]_{t n} \\
& \leq \bar{L}^{+}\left[2^{2 / 3} x n^{2 / 3}\right]_{t n}-\bar{L}^{+}[0]_{t n},
\end{aligned}
$$

while for $x \in[-a, 0]$,

$$
\begin{aligned}
\bar{L}^{-}[0]_{t n}-\bar{L}^{-}\left[2^{2 / 3} x n^{2 / 3}\right]_{t n} & \leq L^{\eta^{(n)}}[0]_{t n}-L^{\eta^{(n)}}\left[2^{2 / 3} x n^{2 / 3}\right]_{t n} \\
& \leq \bar{L}^{+}[0]_{t n}-\bar{L}^{+}\left[2^{2 / 3} x n^{2 / 3}\right]_{t n} .
\end{aligned}
$$

Therefore,

$$
\Delta \bar{H}_{t, n}^{-}(x) \leq \Delta H_{t, n}(x) \leq \Delta \bar{H}_{t, n}^{+}(x), \text { for } x \in[0, a],
$$

and

$$
\Delta \bar{H}_{t, n}^{+}(x) \leq \Delta H_{t, n}(x) \leq \Delta \bar{H}_{t, n}^{-}(x), \text { for } x \in[-a, 0] .
$$

Repeating the same argument, on the event $E_{t, n}^{1 / 2}$ we get that

$$
\Delta \bar{H}_{t, n}^{-}(x) \leq \Delta H_{t, n}^{1 / 2}(x) \leq \Delta \bar{H}_{t, n}^{+}(x), \text { for } x \in[0, a],
$$

and

$$
\Delta \bar{H}_{t, n}^{+}(x) \leq \Delta H_{t, n}^{1 / 2}(x) \leq \Delta \bar{H}_{t, n}^{-}(x), \text { for } x \in[-a, 0] .
$$

Together with (4.2) and (4.3), this implies that, on $E_{t, n} \cap E_{t, n}^{1 / 2}$ we have that

$$
\begin{aligned}
\left|\Delta H_{t, n}(x)-\Delta H_{t, n}^{1 / 2}(x)\right| & \leq\left(\Delta \bar{H}_{t, n}^{+}(x)-\Delta \bar{H}_{t, n}^{-}(x)\right) \mathbf{1}\{x \in[0, a]\} \\
& +\left(\Delta \bar{H}_{t, n}^{-}(x)-\Delta \bar{H}_{t, n}^{+}(x)\right) \mathbf{1}\{x \in[-a, 0]\} \\
& \leq \Delta \bar{H}_{t, n}^{+}(a)-\Delta \bar{H}_{t, n}^{-}(a) \\
& +\Delta \bar{H}_{t, n}^{-}(-a)-\Delta \bar{H}_{t, n}^{+}(-a)
\end{aligned}
$$


and hence,

$$
\left\|\Delta H_{t, n}(\cdot)-\Delta H_{t, n}^{1 / 2}(\cdot)\right\|_{\infty, a}:=\sup _{x \in[-a, a]}\left|\Delta H_{t, n}(x)-\Delta H_{t, n}^{1 / 2}(x)\right| \leq I_{t, n}(a),
$$

where

$$
I_{t, n}(a):=\Delta \bar{H}_{t, n}^{+}(a)-\Delta \bar{H}_{t, n}^{-}(a)+\Delta \bar{H}_{t, n}^{-}(-a)-\Delta \bar{H}_{t, n}^{+}(-a) .
$$

Notice that $2^{4 / 3} n^{1 / 3} I_{t, n}(a)$ is equal to

$$
\left(\bar{L}^{+}\left[2^{2 / 3} a n^{2 / 3}\right]_{t n}-\bar{L}^{+}\left[-2^{2 / 3} a n^{2 / 3}\right]_{t n}\right)-\left(\bar{L}^{-}\left[2^{2 / 3} a n^{2 / 3}\right]_{t n}-\bar{L}^{-}\left[-2^{2 / 3} a n^{2 / 3}\right]_{t n}\right),
$$

and thus, by Lemma 3.4, $I_{t, n}(a) \geq 0$. Therefore,

$$
\begin{aligned}
\mathbb{P}\left(\left\|\Delta H_{t, n}(\cdot)-\Delta H_{t, n}^{1 / 2}(\cdot)\right\|_{\infty, a}>\eta\right) & \leq \mathbb{P}\left(E_{t, n}^{c}\right)+\mathbb{P}\left(\left(E_{t, n}^{1 / 2}\right)^{c}\right)+\mathbb{P}\left(I_{t, n}(a)>\eta\right) \\
& \leq \mathbb{P}\left(E_{t, n}^{c}\right)+\mathbb{P}\left(\left(E_{t, n}^{1 / 2}\right)^{c}\right)+\frac{\mathbb{E}\left(I_{t, n}(a)\right)}{\eta}
\end{aligned}
$$

To control $\mathbb{E}\left(I_{t, n}(a)\right)$, write $2^{4 / 3} n^{1 / 3} I_{t, n}(a)$ as a sum of the increments (3.4):

$$
2^{4 / 3} n^{1 / 3} I_{t, n}(a)=\sum_{k=-\left\lfloor 2^{2 / 3} a n^{2 / 3}\right\rfloor+1}^{\left\lfloor 2^{2 / 3} a n^{2 / 3}\right\rfloor}\left(\zeta_{k+1, n}^{+}-\zeta_{k+1, n}^{-}\right) .
$$

By (3.5),

$$
\begin{aligned}
\mathbb{E}\left(\zeta_{k, n}^{+}-\zeta_{k, n}^{-}\right) & =\left(\frac{1}{1-\rho_{t, n}^{+}}-\frac{1}{1-\rho_{t, n}^{-}}\right)+\left(\frac{1}{\rho_{t, n}^{-}}-\frac{1}{\rho_{t, n}^{+}}\right) \\
& =\left(\frac{1}{\left(1-\rho_{t, n}^{+}\right)\left(1-\rho_{t, n}^{-}\right)}+\frac{1}{\rho_{t, n}^{+} \rho_{t, n}^{-}}\right)\left(\rho_{t, n}^{+}-\rho_{t, n}^{-}\right) \\
& =2 \frac{\rho_{t, n}^{+}-\rho_{t, n}^{-}}{\rho_{t, n}^{+} \rho_{t, n}^{-}}
\end{aligned}
$$

Since $\rho_{t, n}^{ \pm} \rightarrow 1 / 2$, as $n \rightarrow \infty$

$$
0 \leq \mathbb{E}\left(\zeta_{k, n}^{+}-\zeta_{k, n}^{-}\right) \leq 9\left(\rho_{t, n}^{+}-\rho_{t, n}^{-}\right)=18 c \frac{\delta_{t}^{-1 / 4}}{(t n)^{1 / 3}},
$$

for large enough $n$, and this shows that

$$
2^{4 / 3} n^{1 / 3} \mathbb{E}\left(I_{n, t}(a)\right) \leq\left(2 \times 2^{2 / 3} a n^{2 / 3}\right) \times\left(18 c \frac{\delta_{t}^{-1 / 4}}{(t n)^{1 / 3}}\right) \Rightarrow \mathbb{E}\left(I_{n, t}(a)\right) \leq 23 c \sqrt{a} \delta_{t}^{1 / 4},
$$

for large enough $n$, where we use that $a t^{-1 / 3} \delta_{t}^{-1 / 4}=\sqrt{a} \delta_{t}^{1 / 4}$. This finally yields to

$$
\mathbb{P}\left(\left\|\Delta H_{t, n}(\cdot)-\Delta H_{t, n}^{1 / 2}(\cdot)\right\|_{\infty, a}>\eta\right) \leq \mathbb{P}\left(E_{t, n}(r)^{c}\right)+\mathbb{P}\left(E_{t, n}^{1 / 2}(r)^{c}\right)+\frac{\sqrt{a} \delta_{t}^{1 / 4}}{\eta},
$$

for large enough $n$ and $c=23^{-1}$. By Lemma 3.8 (recall that $r_{t}:=\delta_{t}^{-1 / 4}$ ),

$$
\underset{n}{\limsup } \mathbb{P}\left(E_{t, n}(r)^{c}\right)+\mathbb{P}\left(E_{t, n}^{1 / 2}(r)^{c}\right) \leq 4 C_{1} \delta_{t}^{3 / 4}+\phi_{t}\left(\delta_{t}^{-1 / 4}\right)+\phi_{t}^{1 / 2}\left(\delta_{t}^{-1 / 4}\right),
$$

where $\phi_{t}^{1 / 2}$ is the upper bound with respect to initial profile $\mathrm{h}^{1 / 2}$. It converges to Brownian motion, which clearly satisfies (1.6). Recall that we have chosen 
$\left(\mathfrak{h}_{t}(\cdot ; \mathfrak{h}), \mathfrak{h}_{t}(\cdot ; \mathfrak{b})\right)$ as a sub-sequential weak limit point of $\left(H_{t, n}, H_{t, n}^{1 / 2}\right)$. By the previous estimates we can conclude that for such a pair $\left(\mathfrak{h}_{t}(\cdot ; \mathfrak{h}), \mathfrak{h}_{t}(\cdot ; \mathfrak{b})\right)$ we must have that

$$
\mathbb{P}\left(\left\|\Delta \mathfrak{h}_{t}(\cdot ; \mathfrak{h})-\Delta \mathfrak{h}_{t}(\cdot ; \mathfrak{b})\right\|_{\infty, a}>\eta \sqrt{a}\right) \leq \theta\left(\delta_{t}\right)+\frac{\delta_{t}^{1 / 4}}{\eta}
$$

and Proposition 3.6 completes the proof of Theorem 1.1, with $\theta(\delta)=4 C_{1} \delta^{3 / 4}+$ $\phi\left(\delta^{-1 / 4}\right)+\phi^{1 / 2}\left(\delta^{-1 / 4}\right)$.

\section{Proof of Proposition 3.6}

The proof of Proposition 3.6 relies on tail estimates for the location of the maxima in the definition of the last-passage percolation time. Recall that

$$
L^{\eta}(\mathbf{x}):=\max _{k: \mathbf{x}_{k} \leq \mathbf{x}} L\left(\mathbf{x}_{k}, \mathbf{x}\right),
$$

and let $K_{t, n}^{ \pm} \in \mathbb{Z}$ be the index of the location of the maxima for $\mathbf{y}_{t, n}^{ \pm}$, i.e.

$$
L^{\eta}\left(\mathbf{y}_{t, n}^{ \pm}\right)=L\left(\mathbf{x}_{K_{t, n}^{ \pm}}, \mathbf{y}_{t, n}^{ \pm}\right) .
$$

By definition, $Z^{\eta^{(n)}}\left(\mathbf{y}_{t, n}^{ \pm}\right)$belongs to the intersection between the maximal path $\pi^{\eta^{(n)}}\left(\mathbf{y}_{t, n}^{ \pm}\right)$, starting at $\mathbf{x}_{K_{t, n}^{ \pm}}$and ending at $\mathbf{y}_{t, n}^{ \pm}$, and the non-negative coordinate axes. If we are able to control the order of magnitude of $K_{t, n}^{ \pm}$then Proposition 3.6 will follow from well known results on the fluctuations of point to point maximal paths.

Lemma 5.1. Under (1.6) there exist $\psi_{1}(r)$ and $t_{0}>0$ such that for all $t>t_{0}$

$$
\limsup _{n \rightarrow \infty} \mathbb{P}\left(\left|K_{n, t}^{ \pm}\right| \geq r(t n)^{2 / 3}\right) \leq \psi_{1}(r) \text { and } \lim _{r \rightarrow \infty} \psi_{1}(r)=0 .
$$

Let $\mathbf{R}=\mathbf{R}(n, t, r) \subseteq \mathbb{R}^{2}$ be a rectangle centered at the origin and of size of $r(t n)^{2 / 3}$ in the anti-diagonal direction and of size $r(t n)^{1 / 3}$ in the diagonal direction (Figure 5.1). By combining Lemma 5.1 together with (2.4), one has that there exist $t_{0}>0$ and $\psi_{2}(r)$ such that for all $t \geq t_{0}$

$$
\limsup _{n \rightarrow \infty} \mathbb{P}\left(\mathbf{x}_{K_{t, n}^{ \pm}} \in \mathbf{R}\right) \leq \psi_{2}(r) \text {, and } \lim _{r \rightarrow \infty} \psi_{2}(r)=0 .
$$

To conclude the proof of Proposition 3.6, we need to show that for all point $\mathbf{p} \in \mathbf{R}$, that is a corner of $\mathrm{h}$ differently from $(1,1)$, the first intersection (following the upright orientation) between the point-to-point maximal path $\pi(\mathbf{p}, \mathbf{y})\left(\mathbf{y} \equiv \mathbf{y}_{t, n}^{ \pm}\right)$and the non-negative coordinate axes is at distance of order $(t n)^{2 / 3}$ from the origin. Now, let $\mathbf{u}$ and $\mathbf{v}$ be as it is indicated in Figure 5.1. Notice that both $\mathbf{u}$ and $\mathbf{v}$ are at a distance of order $(t n)^{2 / 3}$ from the origin, and that every maximal path starting at some point $\mathbf{p} \in \mathbf{R}$ and ending at $\mathbf{y}$ is below $\pi(\mathbf{u}, \mathbf{y})$ and above $\pi(\mathbf{v}, \mathbf{y})$. Therefore, the intersection of $\pi(\mathbf{p}, \mathbf{y})$ with the non-negative coordinate axes lyes in between the respective intersections of $\pi(\mathbf{u}, \mathbf{y})$ and $\pi(\mathbf{v}, \mathbf{y})$. To prove that these intersections are at a distance of order $(t n)^{2 / 3}$ from the origin one only needs to use upper bounds for fluctuations of point-to-point maximal paths (Theorem 2.5 in Balázs et al., 2006). 


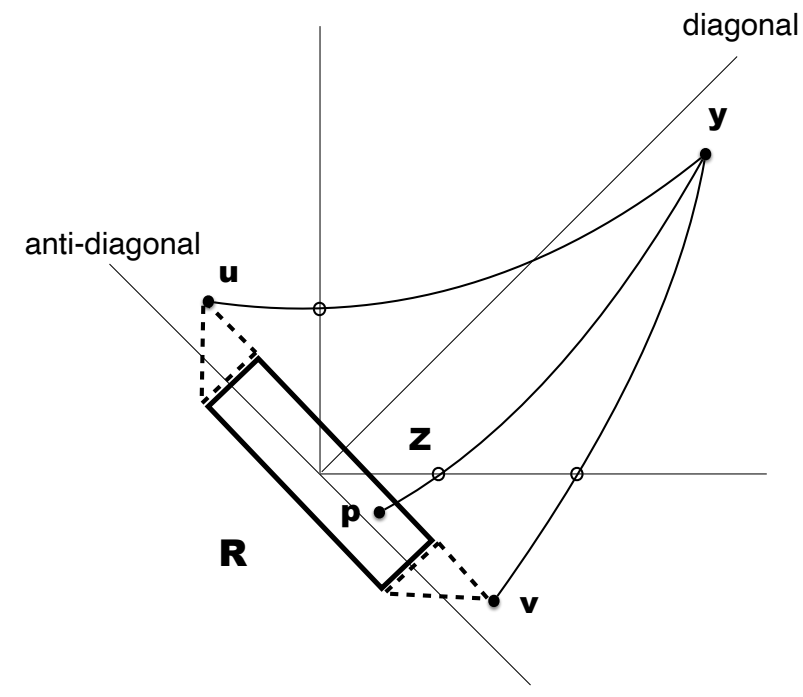

Figure 5.1. Enclosing a maximal path starting at some point $\mathbf{p} \in \mathbf{R}$ by the point-to-point maximal paths starting at $\mathbf{u}$ and $\mathbf{v}$.

Proof of Lemma 5.1 Define

$$
\begin{aligned}
& L_{r}^{\eta}(\mathbf{x}):=\max _{|k|>r n^{2 / 3}: \mathbf{x}_{k} \leq \mathbf{x}} L\left(\mathbf{x}_{k}, \mathbf{x}\right), \\
& H_{t, n}^{r}(x)=\frac{L_{r}^{\eta^{(n)}}\left[2^{2 / 3} x n^{2 / 3}\right]_{\lfloor t n\rfloor}-4 n t}{2^{4 / 3} n^{1 / 3}}
\end{aligned}
$$

and

$$
H_{t, n}^{\mathrm{nw}}(x)=\frac{L\left(\mathbf{0},\left[2^{2 / 3} x n^{2 / 3}\right]_{\lfloor t n\rfloor}\right)-4 n t}{2^{4 / 3} n^{1 / 3}} .
$$

Thus

$$
\begin{aligned}
\mathbb{P}\left(\left|K_{t, n}^{ \pm}\right|>r t^{2 / 3} n^{2 / 3}\right) & \leq \mathbb{P}\left(L\left(\mathbf{0}, \mathbf{y}_{t, n}^{ \pm}\right)<L_{r t^{2 / 3}}^{\eta^{(n)}}\left(\mathbf{y}_{t, n}^{ \pm}\right)\right) \\
& =\mathbb{P}\left(H_{t, n}^{\mathrm{nw}}\left( \pm t^{2 / 3}\right)<H_{t, n}^{r t^{2 / 3}}\left( \pm t^{2 / 3}\right)\right)
\end{aligned}
$$

since $L\left(\mathbf{0}, \mathbf{y}_{t, n}^{ \pm}\right) \leq L^{\eta^{(n)}}\left(\mathbf{y}_{t, n}^{ \pm}\right)$and $L^{\eta}\left(\mathbf{y}_{t, n}^{ \pm}\right)=L_{r}^{\eta^{(n)}}\left(\mathbf{y}_{t, n}^{ \pm}\right)$if $\left|K_{t, n}^{ \pm}\right|>r(t n)^{2 / 3}$. The LPP time $L\left(\mathbf{0},\left[2^{2 / 3} x n^{2 / 3}\right]_{\lfloor t n\rfloor}\right)$ corresponds to the narrow wedge initial profile (Johansson, 2003; Matetski et al., 2016) and

$$
\lim _{n \rightarrow \infty} H_{t, n}^{\mathrm{nw}}(x) \stackrel{\text { dist. }}{=} t^{1 / 3} A\left(x t^{-2 / 3}\right)-\frac{x^{2}}{t}
$$

while for $L_{r t^{2 / 3}}^{\eta^{(n)}}\left(\mathbf{y}_{t, n}^{ \pm}\right)$we have (by (1.5))

$$
\lim _{n \rightarrow \infty} H_{t, n}^{r t^{2 / 3}}(x) \stackrel{\text { dist. }}{=} \mathfrak{h}_{t}^{r t^{2 / 3}}(x ; \mathfrak{h}) \stackrel{\text { dist. }}{=} \sup _{|z|>r t^{2 / 3}}\left\{\mathfrak{h}(z)+t^{1 / 3} A\left(z t^{-2 / 3}\right)-\frac{(x-z)^{2}}{t}\right\} .
$$


Since for any $R>0$,

$$
\begin{aligned}
& \mathbb{P}\left(H_{t, n}^{\mathrm{nw}}\left( \pm t^{2 / 3}\right)<H_{t, n}^{r t^{2 / 3}}\left( \pm t^{2 / 3}\right)\right) \\
& \leq \mathbb{P}\left(H_{t, n}^{\mathrm{nw}}\left( \pm t^{2 / 3}\right) \leq-R\right)+\mathbb{P}\left(-R<H_{t, n}^{r t^{2 / 3}}\left( \pm t^{2 / 3}\right)\right),
\end{aligned}
$$

by (5.2), we get that

$$
\begin{aligned}
& \underset{n}{\limsup } \mathbb{P}\left(\left|K_{t, n}^{ \pm}\right|>r(t n)^{2 / 3}\right) \\
& \leq \mathbb{P}\left(t^{1 / 3}(A(1)-1) \leq-R\right)+\mathbb{P}\left(-R<\mathfrak{h}_{t}^{r t^{2 / 3}}\left( \pm t^{2 / 3} ; \mathfrak{h}\right)\right) .
\end{aligned}
$$

By using (5.3) with $R=t^{1 / 3} r^{2} / 8$ we have that

$$
\begin{aligned}
& \limsup _{n} \mathbb{P}\left(\left|K_{t, n}^{ \pm}\right|>r(t n)^{2 / 3}\right) \\
& \leq \mathbb{P}\left(A(1) \leq-\frac{r^{2}}{8}+1\right)+\mathbb{P}\left(-t^{1 / 3} \frac{r^{2}}{8}<\mathfrak{h}_{t}^{r t^{2 / 3}}\left( \pm t^{2 / 3} ; \mathfrak{h}\right)\right) .
\end{aligned}
$$

Since (by stationarity of $A$ )

$$
\mathbb{P}\left(A(1) \leq-\frac{r^{2}}{8}+1\right)=\mathbb{P}\left(A(0) \leq-\frac{r^{2}}{8}+1\right) \rightarrow 0, \text { as } r \rightarrow \infty,
$$

we only need to show that

$$
\mathbb{P}\left(-t^{1 / 3} \frac{r^{2}}{8}<\mathfrak{h}_{t}^{r t^{2 / 3}}\left( \pm t^{2 / 3} ; \mathfrak{h}\right)\right) \rightarrow 0, \text { as } r \rightarrow \infty
$$

uniformly on $t$. Indeed, for $\gamma_{t}=t^{1 / 3}$, we note that

$$
\begin{aligned}
\mathfrak{h}_{t}^{r t^{2 / 3}}\left( \pm t^{2 / 3} ; \mathfrak{h}\right) & \stackrel{\text { dist. }}{=} \sup _{|z|>r t^{2 / 3}}\left\{\mathfrak{h}(z)+t^{1 / 3} A\left(z t^{-2 / 3}\right)-\frac{\left(z- \pm t^{2 / 3}\right)^{2}}{t}\right\} \\
& =t^{1 / 3} \sup _{|z|>r t^{2 / 3}}\left\{t^{-1 / 3} \mathfrak{h}(z)+A\left(z t^{-2 / 3}\right)-\left(z t^{-2 / 3}- \pm 1\right)^{2}\right\} \\
& =t^{1 / 3} \sup _{|u|>r}\left\{S_{\gamma_{t}} \mathfrak{h}(u)+A(u)-(u- \pm 1)^{2}\right\} \\
& \leq t^{1 / 3} \sup _{|u|>r}\left\{S_{\gamma_{t}} \mathfrak{h}(u)+A(u)-\frac{u^{2}}{2}\right\},
\end{aligned}
$$

for large enough $r>0$. Recall (1.2) and notice that if $S_{\gamma_{t}} \mathfrak{h}(u) \leq \frac{r}{8}|u|$ then $S_{\gamma_{t}} \mathfrak{h}(u) \leq$ $\frac{u^{2}}{4}$ for $|u|>r>1$, and hence

$$
\mathbb{P}\left(-t^{1 / 3} \frac{r^{2}}{8}<\mathfrak{h}_{t}^{r t^{2 / 3}}\left( \pm t^{2 / 3} ; \mathfrak{h}\right)\right) \leq \psi(r / 8)+\mathbb{P}\left(-\frac{r^{2}}{8}<\sup _{|u|>r}\left\{A(u)-\frac{u^{2}}{4}\right\}\right)
$$

By (b)-Proposition 2.13 Corwin et al. (2016),

$$
\mathbb{P}\left(-\frac{r^{2}}{8}<\sup _{|u|>r}\left\{A(u)-\frac{u^{2}}{4}\right\}\right) \rightarrow 0, \text { as } r \rightarrow \infty
$$




\section{Acknowledgements}

The author would like to thank Patrik Ferrari and Daniel Remenik for useful comments and enlightening discussions concerning the subject, and an anonymous referee for pointing out several mistakes in a preliminary version. This research was supported in part by the International Centre for Theoretical Sciences (ICTS) during a visit for participating in the program - Universality in random structures: Interfaces, Matrices, Sandpiles (Code: ICTS/urs2019/01).

\section{References}

Amir, G., Corwin, I., and Quastel, J. Probability distribution of the free energy of the continuum directed random polymer in $1+1$ dimensions. Comm. Pure Appl. Math., 64 (4), 466-537 (2011). MR2796514.

Baik, J., Ferrari, P. L., and Péché, S. Limit process of stationary TASEP near the characteristic line. Comm. Pure Appl. Math., 63 (8), 1017-1070 (2010). MR2642384.

Baik, J. and Rains, E. M. Limiting distributions for a polynuclear growth model with external sources. J. Statist. Phys., 100 (3-4), 523-541 (2000). MR1788477.

Balázs, M., Cator, E., and Seppäläinen, T. Cube root fluctuations for the corner growth model associated to the exclusion process. Electron. J. Probab., 11, no. 42, 1094-1132 (2006). MR2268539.

Billingsley, P. Convergence of probability measures. John Wiley and Sons, Inc., New York-London-Sydney (1968). MR0233396.

Borodin, A., Corwin, I., Ferrari, P., and Vető, B. Height fluctuations for the stationary KPZ equation. Math. Phys. Anal. Geom., 18 (1), Art. 20, 95 (2015). MR3366125.

Borodin, A., Ferrari, P. L., Prähofer, M., and Sasamoto, T. Fluctuation properties of the TASEP with periodic initial configuration. J. Stat. Phys., 129 (5-6), 1055-1080 (2007). MR2363389.

Cator, E. and Pimentel, L. P. R. On the local fluctuations of last-passage percolation models. Stochastic Process. Appl., 125 (2), 538-551 (2015). MR3293293.

Corwin, I. and Hammond, A. Brownian Gibbs property for Airy line ensembles. Invent. Math., 195 (2), 441-508 (2014). MR3152753.

Corwin, I., Liu, Z., and Wang, D. Fluctuations of TASEP and LPP with general initial data. Ann. Appl. Probab., 26 (4), 2030-2082 (2016). MR3543889.

Corwin, I., Quastel, J., and Remenik, D. Renormalization fixed point of the KPZ universality class. J. Stat. Phys., 160 (4), 815-834 (2015). MR3373642.

D. Dauvergne, J. O. and Virág, B. The directed landscape. ArXiv Mathematics e-prints (2018). arXiv: 1812.00309.

Ferrari, P. L. and Occelli, A. Universality of the GOE Tracy-Widom distribution for TASEP with arbitrary particle density. Electron. J. Probab., 23, Paper No. 51, 24 (2018). MR3814245.

Hägg, J. Local Gaussian fluctuations in the Airy and discrete PNG processes. Ann. Probab., 36 (3), 1059-1092 (2008). MR2408583.

Hammond, A. A patchwork quilt sewn from Brownian fabric: regularity of polymer weight profiles in Brownian last passage percolation. Forum Math. Pi, 7, e2, 69 (2019). MR3987302. 
Johansson, K. Shape fluctuations and random matrices. Comm. Math. Phys., 209 (2), 437-476 (2000). MR1737991.

Johansson, K. Discrete polynuclear growth and determinantal processes. Comm. Math. Phys., 242 (1-2), 277-329 (2003). MR2018275.

Kardar, M., Parisi, G., and Zhang, Y.-C. Dynamic Scaling of Growing Interfaces. Phys. Rev. Lett., 56, 889-892 (1986). DOI: 10.1103/PhysRevLett.56.889.

Matetski, K., Quastel, J., and Remenik, D. The KPZ fixed point. ArXiv Mathematics e-prints (2016). arXiv: 1701.00018.

Pimentel, L. P. R. Local behaviour of airy processes. J. Stat. Phys., 173 (6), 1614-1638 (2018). MR3880997.

Prähofer, M. and Spohn, H. Scale invariance of the PNG droplet and the Airy process. J. Statist. Phys., 108 (5-6), 1071-1106 (2002). MR1933446.

Sasamoto, T. Spatial correlations of the 1D KPZ surface on a flat substrate. $J$. Phys. A, 38 (33), L549-L556 (2005). MR2165697.

Seppäläinen, T. and Shen, X. Coalescence estimates for the corner growth model with exponential weights. Electron. J. Probab., 25, Paper No. 85, 31 (2020). MR4125790. 